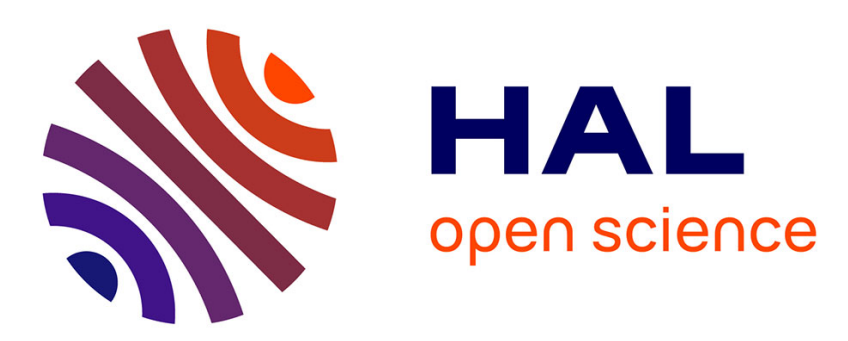

\title{
Intérêts et difficultés de la mise en ouvre du traitement thermique des matériaux métalliques par lasers
}

\author{
J. Merlin, J. Dietz
}

\section{To cite this version:}

J. Merlin, J. Dietz. Intérêts et difficultés de la mise en œuvre du traitement thermique des matériaux métalliques par lasers. Revue de Physique Appliquée, 1987, 22 (8), pp.735-745. 10.1051/rphysap:01987002208073500 . jpa-00245603

\section{HAL Id: jpa-00245603 https://hal.science/jpa-00245603}

Submitted on 1 Jan 1987

HAL is a multi-disciplinary open access archive for the deposit and dissemination of scientific research documents, whether they are published or not. The documents may come from teaching and research institutions in France or abroad, or from public or private research centers.
L'archive ouverte pluridisciplinaire HAL, est destinée au dépôt et à la diffusion de documents scientifiques de niveau recherche, publiés ou non, émanant des établissements d'enseignement et de recherche français ou étrangers, des laboratoires publics ou privés. 
Classification

Physics Abstracts

$42.55 \mathrm{R}-44.90-61.80 \mathrm{~B}-78.90-81.40$

\title{
Intérêts et difficultés de la mise en œuvre du traitement thermique des matériaux métalliques par lasers
}

\author{
J. Merlin et J. Dietz \\ C.A.L.F.E.T. MAT., G.E.M.P.P.M., U.A. 341, I.N.S.A. Bât. 502, 69621 Villeurbanne Cedex, France
}

(Reçu le 27 octobre 1986, révisé le 22 avril 1987, accepté le 12 mai 1987)

Article de mise au point.

\begin{abstract}
Résumé. - Les projets d'applications des lasers en traitement thermique des matériaux métalliques sont de plus en plus nombreux mais pas toujours réalistes ; on a donc essayé de faire le point sur les possibilités et les limitations de cette technologie. Pour cela, on est parti des caractéristiques des sources existantes, des théories élémentaires concernant les phénomènes de transfert thermique et les transformations microstructurales. Il en ressort qu'un nombre restreint de sources sont utilisables pour ce type d'applications et que les paramètres de traitement (puissance, temps d'interaction, dimension du faisceau) ne peuvent être choisis que dans une gamme très étroite, quelle que soit la nature de la transformation microstructurale recherchée et l'alliage retenu. En outre, l'interdépendance inéluctable des paramètres effectifs de traitement (température, temps de maintien, vitesses de chauffage et de refroidissement) demande une bonne connaissance des phénomènes métallurgiques élémentaires et une grande maîtrise des caractéristiques de la source. Cette maîtrise passe par la réalisation d'installations plus sophistiquées que celles actuellement existantes dans l'industrie pour les applications de type découpe et soudage. Elle nécessite en particulier le suivi en temps réel des caractéristiques du faisceau (puissance, répartition d'énergie) et des effets induits (température de surface atteinte...) et la régulation de la source à partir de ces grandeurs.
\end{abstract}

\begin{abstract}
Laser application projects in metallic materials heat treatment are more and more numerous but they are not always realistic. Therefore it has been tried to take stock of capacities and limits of this technology. With this intention, existing source characteristics and elementary heat conduction and microstructural transformations theories have been considered. Issuing from these, only a short number of sources are fit for use in this type of applications, and working parameters (power, interaction time and beam size) may be chosen in a very short range, whatever the nature of the microstructural transformation and the alloy are. Furthermore, the compulsory interdependence between actual treatment parameters (temperature, duration, heating and cooling rates) needs a real knowledge of the metallurgical elementary phenomena and a true control of the source characteristics. This control passes through the realization of much more sophisticated devices than those presently existing for welding and cutting in manufacturing. Particularly real time measurements of beam characteristics (power and energy distribution) induced effects (reached surface temperature, and others) and the source control by them are necessary.
\end{abstract}

\section{Introduction.}

Dans de nombreux domaines on évoque des possibilités d'application des lasers ; si certains projets sont tout à fait réalistes, d'autres sont parfaitement utopiques. Cette situation tient certainement à l'aspect novateur de la technologie laser mais sans doute aussi au caractère quasi magique que présente la lumière dans l'inconscient collectif. Le domaine des traitements thermiques des matériaux ne fait pas exception à la règle; les projets d'applications des lasers y sont nombreux et bien que les principes de base gouvernant ces traitements aient été établis depuis une dizaine d'années au moins, comme nous le verrons dans la suite de cet article, des idées plus ou moins erronées continuent de circuler. Il nous est donc apparu intéressant de faire le point sur les possibilités réelles et les contraintes de cette technologie dans ce champ d'applications afin d'éviter des déconvenues ultérieures.

Pour cela, nous commencerons par rappeler brièvement que le but du traitement thermique d'un 
matériau est de réaliser plus ou moins localement une modification microstructurale par communication d'une quantité d'énergie thermique d'autant plus grande que le volume concerné est important. La métallurgie physique enseigne que la température atteinte, le temps de maintien ainsi que les vitesses de variation de la température sont des paramètres qui conditionneront la nature de la transformation réalisée.

Les lasers utilisés en tant que source d'énergie pour réaliser un traitement thermique devront donc :

1) communiquer une quantité d'énergie au matériau suffisante pour induire une transformation dans un volume donné,

2) permettre de moduler les températures et les temps de traitement.

L'analyse détaillée de ces impératifs va nous permettre :

a) de définir parmi les nombreuses sources laser existantes celles qui présentent un intérêt vis-à-vis des traitements thermiques,

b) de déterminer le mode opératoire permettant d'aboutir à une transformation microstructurale de caractéristiques données,

c) de préciser les différents traitements thermiques susceptibles d'être réalisés,

d) de définir les caractéristiques de l'outil laser idéal en traitement thermique.

\section{Les sources laser utilisables pour le traitement thermique des matériaux.}

Nous nous intéresserons ici aux applications de type métallurgique ou mécanique donc se rapportant essentiellement à des alliages métalliques se présentant sous forme de pièces ayant des dimensions de l'ordre de 1 à $100 \mathrm{~mm}$. Les volumes concernés par les transformations sont habituellement de l'ordre $\mathrm{du} \mathrm{cm}^{3}$ ou plus. Or, la chaleur spécifique par unité de volume $c_{v}^{+}$pour les différents matériaux de cette classe reste toujours de l'ordre de $3 \mathrm{~J} . \mathrm{cm}^{-3} \cdot \mathrm{K}^{-1}$, voir tableau I, [1]. Si la transformation a lieu à l'état solide, l'accroissement de température sera d'environ $1000{ }^{\circ} \mathrm{C}$ et il sera donc nécessaire de communiquer environ $3 \mathrm{~kJ} . \mathrm{cm}^{-3}$. Si la transformation nécessite le passage à l'état liquide il faut en outre prendre

Tableau I. - Caractéristiques thermiques moyennes à l'état solide d'éléments métalliques pris parmi les métaux les plus usuels (caractère thermique « conducteur 》, $\mathrm{C}$; « normal », N; « isolant », I) (d'après [1] et [2]).

[Average solid state thermal characteristics of several common metals (good heat conductor, $\mathrm{C}$; average, $\mathrm{N}$; poor, I) [1] and [2].]

\begin{tabular}{|c|c|c|c|c|c|c|c|}
\hline Elément & $\begin{array}{c}c_{\mathrm{v}}^{+} \\
\left(\mathrm{J} . \mathrm{cm}^{-3} \cdot \mathrm{K}^{-1}\right)\end{array}$ & $\begin{array}{c}k \\
\left(\mathrm{~W} \cdot \mathrm{mm}^{-1} \cdot \mathrm{K}^{-1}\right)\end{array}$ & $\begin{array}{c}\alpha \\
\left(\mathrm{mm}^{2} \cdot \mathrm{s}^{-1}\right)\end{array}$ & $\begin{array}{c}\sqrt{\alpha} / k \\
\left(\mathrm{~W}^{-1} \cdot \mathrm{mm}^{2} \cdot \mathrm{K} \cdot \mathrm{s}^{1 / 2}\right)\end{array}$ & $\begin{array}{c}T_{\mathrm{F}} \\
(\mathrm{K})\end{array}$ & $L_{\mathrm{F}} /\left(T_{\mathrm{F}} \cdot c_{\mathrm{v}}^{+}\right)$ & Caractère \\
\hline $\mathrm{Al}$ & 2,6 & 0,22 & 85 & 42 & 933 & 0,43 & $\mathrm{C}$ \\
\hline $\mathrm{Au}$ & 2,5 & 0,3 & 120 & 36 & 1336 & 0,37 & $\mathrm{C}$ \\
\hline $\mathrm{Cr}$ & 3,3 & 0,067 & 20 & 67 & 2148 & 0,42 & $\mathrm{~N}$ \\
\hline $\mathrm{Cu}$ & 3,45 & 0,39 & 115 & 28 & 1356 & 0,38 & $\mathrm{C}$ \\
\hline $\mathrm{Fe}$ & 3,6 & 0,075 & 20 & 60 & 1809 & 0,33 & $\mathrm{~N}$ \\
\hline $\mathrm{Mg}$ & 1,8 & 0,15 & 85 & 61 & 923 & 0,43 & $\mathrm{~N}$ \\
\hline $\mathrm{Ni}$ & 3,9 & 0,092 & 25 & 54 & 1726 & 0,41 & $\mathrm{~N}$ \\
\hline $\mathrm{Pb}$ & 1,45 & 0,035 & 25 & 143 & 600 & 0,33 & $\mathrm{I}$ \\
\hline $\mathrm{Ti}$ & 2,3 & 0,017 & 10 & 185 & 1941 & 0,42 & $\mathrm{I}$ \\
\hline $\mathrm{W}$ & 2,7 & 0,17 & 60 & 45 & 3683 & 0,35 & $\mathrm{C}$ \\
\hline $\mathrm{Zn}$ & 2,7 & 0,11 & 40 & 57 & 692 & 0,36 & $\mathrm{~N}$ \\
\hline $\mathrm{Zr}$ & 1,85 & 0,021 & 10 & 150 & 2125 & 0,42 & $\mathrm{I}$ \\
\hline $\mathrm{Comportement}$ & 3 & 0,1 & 50 & 60 & -1 & 0,4 & \\
\hline moyen & & & & & & \\
\hline
\end{tabular}


en compte la chaleur latente de fusion $L_{\mathrm{F}}$, qui, pour les différents éléments $[1,2]$, reste d'un point de vue énergétique équivalente à un accroissement de température à l'état solide de $0,4 T_{\mathrm{F}}\left(L_{\mathrm{F}} / c_{\mathrm{v}}^{+} \simeq 0,4 T_{\mathrm{F}}\right.$ avec $T_{\mathrm{F}}$ : température de fusion en $\mathrm{K}$, voir Tab. I). Selon le niveau maximum de température atteint (environ $2000^{\circ} \mathrm{C}$ en général) il faudra donc communiquer 2 à 3 fois plus d'énergie que dans le cas précédent. Le traitement de $1 \mathrm{~cm}^{3} \cdot \mathrm{s}^{-1}$ d'un alliage à l'état solide nécessite donc de communiquer au matériau environ $3 \mathrm{~kW}$ et le passage à l'état liquide au moins $6 \mathrm{~kW}$, valeurs qu'il est nécessaire de majorer pratiquement d'un coefficient de l'ordre de deux pour tenir compte des pertes dans le transport du faisceau et du rendement de conversion de l'énergie photonique en énergie thermique.

En d'autres termes une source émettant en continu quelques $\mathrm{kW}$ permettra de réaliser chaque seconde une transformation microstructurale s'étendant sur une surface de $1 \mathrm{~cm}^{2}$ et une profondeur de l'ordre du millimètre, tandis qu'une source de quelques watts ne permettra de réaliser cette transformation que sur une profondeur de l'ordre du dixième de millimètre.

Pour les applications de type métallurgique, étant donné les caractéristiques géométriques des pièces traitées, cela implique l'usage de sources laser émettant une puissance moyenne de $100 \mathrm{~W}$ à $10 \mathrm{~kW}$ et la réalisation de traitements ayant un caractère superficiel ou localisé.

Cet impératif énergétique entraîne qu'à l'heure actuelle les traitements thermiques ne peuvent être réalisés qu'à l'aide des catégories de sources suivantes dont nous rappelons quelques caractéristiques moyennes [3] :

- laser à gaz $\mathrm{CO}_{2}$ continu $100 \mathrm{~W}-20 \mathrm{~kW}(\lambda=$ $10,6 \mu \mathrm{m})$,

- laser à gaz $\mathrm{CO}_{2}$ pulsé TEA, durée de pulse inférieure à $10^{-4} \mathrm{~s}$ env., fréquence d'émission jusqu'à $1 \mathrm{kHz}$ env. et puissance moyenne inférieure au $\mathrm{kW}(\lambda=10,6 \mu \mathrm{m})$,

- laser à solide YAG pulsé relaxé, de manière à avoir des durées de pulse de $10^{-4}$ à $10^{-2} \mathrm{~s}$, fréquence d'émission jusqu'à $100 \mathrm{~Hz}$ env. et puissance moyenne inférieure au $\mathrm{kW}(\lambda=1,06 \mu \mathrm{m})$.

Ces sources émettent toutes dans l'infrarouge, tout comme les lasers verre-Nd pulsés qui peuvent actuellement aussi être utilisés, ce qui pose certains problèmes vis-à-vis de l'absorption du rayonnement, la réflectivité des surfaces métalliques étant élevée dans ce domaine de longueur d'onde. Les lasers excimères émettant dans l'ultraviolet $(\lambda \approx 0,2 \mu \mathrm{m})$ peuvent donc présenter un intérêt, puisqu'ils paraissent susceptibles dans un proche avenir de délivrer des puissances moyennes de l'ordre de plusieurs centaines de watts.

Remarquons cependant que pour des transformations dans des matériaux à usage micro-électronique ou opto-électronique les volumes concernés étant plusieurs ordres de grandeurs plus faibles, des sources de puissance moyenne nettement plus réduite (de l'ordre du W) et émettant dans le visible peuvent être utilisées (par exemple le laser à argon émettant en continu de l'ordre de $10 \mathrm{~W}-\lambda \approx 0,5 \mu \mathrm{m}$ ).

\section{Mode opératoire pour la réalisation d'une trans- formation donnée.}

\subsection{DISTINCTION DE DIFFÉRENTS RÉGIMES THERMI-} QUES. - Pour le moment nous n'avons considéré que la quantité d'énergie à communiquer globalement au matériau pour réaliser une transformation microstructurale. Bien évidemment la réalité de la transformation et ses caractéristiques sont fonction de la manière dont cette énergie est communiquée, c'est-à-dire, en particulier de la température atteinte et du temps mis pour l'atteindre.

Une réponse précise à ces questions nécessite la prise en compte des problèmes de conversion d'énergie et de transfert thermique. Il est cependant possible de donner des tendances générales du comportement d'un matériau à partir de quelques considérations simples.

$\mathrm{Si}$ nous considérons un faisceau photonique de longueur d'onde $\lambda$ et de section $r^{2}$ transportant une puissance $P_{0}$ et éclairant durant un temps $\tau$ un matériau de conductivité thermique $k$ et de diffusivité thermique $\alpha\left(\alpha=k / c_{v}^{+}\right)$, ce matériau est soumis à une densité de puissance $p_{0}=P_{0} / r^{2}$ mais n'absorbe qu'une fraction $\rho$ du flux incident et la densité de puissance incidente convertie en flux thermique s'exprimera par $p=\rho P_{0} / r^{2}$. Le paramètre $\rho$, usuellement appelé coefficient d'absorption, est faible dans le domaine infrarouge pour les solides métalliques ( 0,1 environ), mais passe à $0,5-0,7$ si des revêtements absorbants du type graphite pulvérisé sont déposés, voire 0,9 dès que l'on atteindra l'état liquide [4]).

En fait, cette absorption du rayonnement s'effectue dans une couche superficielle d'épaisseur $i$ fonction de $\lambda$ et de la nature du matériau [5]. En général, $i$ est d'autant plus faible que $\lambda$ est grand et le matériau conducteur. Pour $\lambda \simeq 10 \mu \mathrm{m}, i \simeq 100 \mathrm{~nm}$ pour les métaux et quelques dizaines de $\mu \mathrm{m}$ pour les matériaux isolants tels que le papier [6], mais $i$ atteint le $\mu \mathrm{m}$ pour les métaux éclairés par un rayonnement U.V.

Si la distance de propagation de la chaleur $s$ durant le temps de l'irradiation (définie par $s=$ $2 \sqrt{\alpha \tau}$ ) est inférieure à $i$, c'est-à-dire si $\tau \leqslant i^{2} / 4 \alpha$, l'énergie incidente restera confinée dans la zone d'interaction. Le régime sera qualifié de « calorimétrique " et l'accroissement local de température $\Delta T$ s'exprimera [6] :

$$
\Delta T \propto(p / i)(\alpha / k) \tau .
$$


Il faut cependant remarquer que ce régime n'intervient pour les matériaux métalliques que si $\tau \leqslant$ $10^{-9} \mathrm{~s}$ et n'est donc pas à considérer pour les traitements thermiques usuels.

Si $\tau \gg i^{2} / 4 \alpha$, l'énergie peut être considérée comme déposée en surface du matériau et cette fois ce sont les dimensions géométriques de l'objet traité (longueur $L$, largeur $l$, épaisseur $h$ ) et du faisceau $(r)$ qui conditionneront les échanges thermiques. Nous citerons les principales situations pouvant être rencontrées (voir en particulier [7]) :

a) $r \geqslant L, l, h$ : cas de pièces de petit volume isolées thermiquement ; on reste constamment dans un régime de type calorimétrique avec une température homogène en volume dès que $\tau \geqslant L^{2} / 4 \alpha$; dans ce cas,

$$
\Delta T \propto\left(\rho P_{0} / L l h\right)(\alpha / k) \tau .
$$

b) $r \geqslant h$ et $L, l>r$ : cas de plaques minces d'étendue infinie; dans ce régime, dès que $\tau \geqslant h^{2} / 4 \alpha$, la température tendra à être homogène dans toute l'épaisseur avec une évolution en $\ln (\tau)$ [8].

c) $L, l, h \gg r$ : cas du solide semi-infini correspondant au traitement superficiel de pièces massives ou d'épaisseur réduite avec couplage thermique sur un support conducteur; cette situation recouvre la plupart des traitements thermiques pouvant être réalisés par laser, nous l'analyserons donc plus en détail et comme cela a été montré [9], deux nouveaux régimes sont à distinguer :

i) si la distance de propagation $s$ durant le temps de l'irradiation est supérieure à $r$, un régime stationnaire tend à s'établir et l'accroissement de la température de surface $\Delta T_{\mathrm{s}}$ est indépendant du temps d'irradiation $\left(\Delta T_{s} \propto P_{0} / k r\right)$. Ce régime présente assez peu d'intérêt du point de vue traitement laser et n'est à prendre en considération que si $\tau>\tau_{c}$ avec $\tau_{\mathrm{c}}=r^{2} / 4 \alpha$.

Remarquons que plus le faisceau est concentré et plus le matériau a une diffusivité thermique élevée, plus cette situation devient probable. Ainsi, pour $r=0,1 \mathrm{~mm}$ et $\alpha \simeq 100 \mathrm{~mm}^{2} \cdot \mathrm{s}^{-1}$ (cas de $\mathrm{Al} \mathrm{ou} \mathrm{Cu}$ ), $\tau_{\mathrm{c}}=2,5 \times 10^{-4} \mathrm{~s}$; par contre avec $r=3 \mathrm{~mm}$ et $\alpha=10 \mathrm{~mm}^{2} \cdot \mathrm{s}^{-1}$ (cas du Ti ou d'aciers inoxydables) $\tau_{\mathrm{c}} \simeq 1 \mathrm{~s}$.

ii) si la condition $\tau<\tau_{c}$ est respectée, on est dans un régime « transitoire » pour lequel l'accroissement de température maximal $\Delta T_{\mathrm{s}}$ observé en surface s'exprime par:

$$
\Delta T_{\mathrm{s}}=A \cdot p \cdot(\sqrt{\alpha} / K) \cdot \sqrt{\tau} .
$$

Le coefficient de proportionnalité $A$ est fonction de la répartition énergétique dans le faisceau mais reste toujours de l'ordre de 1 pour des répartitions courantes de type gaussienne ou homogène cylindrique.
Le rapport $\sqrt{\alpha / k}$ (inverse de l'effusivité thermique du matériau) est caractéristique du comportement thermique en régime transitoire. Ce rapport (voir Tab. I), de l'ordre de $60 \mathrm{~mm}^{2} \cdot \mathrm{s}^{1 / 2} \cdot \mathrm{K} . \mathrm{J}^{-1}$ pour les matériaux thermiquement «normaux» (fer et aciers faiblement alliés) doit être divisé par deux pour les matériaux «bons conducteurs » $(\mathrm{Al}, \mathrm{Cu}$, $\mathrm{Ag}, \mathrm{Au}$, et leurs alliages) et multiplié par deux pour les matériaux mauvais conducteurs, « isolants » $(\mathrm{Ti}$, $\mathrm{Zr}$ et alliages, graphite, aciers inoxydables) [1, 2].

Pour les matériaux métalliques, le régime transitoire s'étend pratiquement de $10^{-9}$ à quelque $10^{-1} \mathrm{~s}$, et tend par conséquent à couvrir toute la gamme des traitements thermiques laser. Il sera donc possible de ne prendre en compte que ce régime pour évaluer les comportements probables des matériaux et définir l'ordre de grandeur des paramètres de traitement.

3.2 PRÉVISION DES COMPORTEMENTS À PARTIR DU RÉGIME TRANSITOIRE. - D'après les valeurs de $\sqrt{\alpha} / k$, et toutes choses étant égales par ailleurs, en particulier le coefficient d'absorption du rayonnement, l'atteinte d'une même température de surface sur des alliages métalliques différents nécessitera de se situer dans une fourchette de densité de puissance variant dans un rapport de 1 à 4 [10]. Il faudra ainsi environ 4 fois plus de puissance pour porter la surface d'une pièce en cuivre à la même température que la surface d'une pièce en titane.

La relation (1) implique également que la réalisation d'un traitement thermique, donc l'atteinte d'une certaine température, nécessite une densité de puissance directement reliée au temps d'interaction $\tau$ entre le rayonnement et la pièce :

$p=A^{\prime} \cdot(k / \sqrt{\alpha}) \cdot \Delta T_{\mathrm{s}} / \sqrt{\tau}$, soit approximativement $\Delta T_{\mathrm{s}} / \sqrt{\tau}$ si on exprime $p$ en W. $\mathrm{cm}^{-2}$.

Ainsi, pour un alliage à comportement thermique « normal» du type acier au carbone, l'atteinte de $1000^{\circ} \mathrm{C}$ en surface, nécessitera pour un temps d'interaction de $10^{-2} \mathrm{~s}$, environ $10^{4} \mathrm{~W} . \mathrm{cm}^{-2}$ tandis que pour $\tau=10^{-6} \mathrm{~s}$, il faudra $1 \mathrm{MW} \cdot \mathrm{cm}^{-2}$. Par conséquent, dans la représentation $\log p=f(\log \tau)$ (Figs. 1 et 2) une isotherme se traduira par une droite de pente $-1 / 2$, tandis que les iso-densités d'énergie («fluence»): $q\left(\mathrm{~J} . \mathrm{cm}^{-2}\right)$ correspondront à des droites de pente - 1. L'atteinte d'une même température de surface nécessitera donc d'autant moins d'énergie que le temps d'interaction sera faible ; il ne faut cependant pas se méprendre sur cette constatation qui a pour corollaire que l'étendue de la transformation sera d'autant plus limitée. Il est possible d'en avoir une idée en introduisant le paramètre d'intensité de transformation $S=q / c_{\mathrm{v}}^{+}$ ( $\simeq q / 3 \mathrm{~cm} . \mathrm{K}$ pour la plupart des matériaux) ; ainsi, $1 \mathrm{~kJ} . \mathrm{cm}^{-2}$ permettra de porter une épaisseur de l'ordre de $1 \mathrm{~cm}$ à $300{ }^{\circ} \mathrm{C}$ ou $1 \mathrm{~mm}$ à $3000^{\circ} \mathrm{C}$ tandis que $1 \mathrm{~J} . \mathrm{cm}^{-2}$ ne permettra de porter que $1 / 100 \mathrm{~mm}$ à 


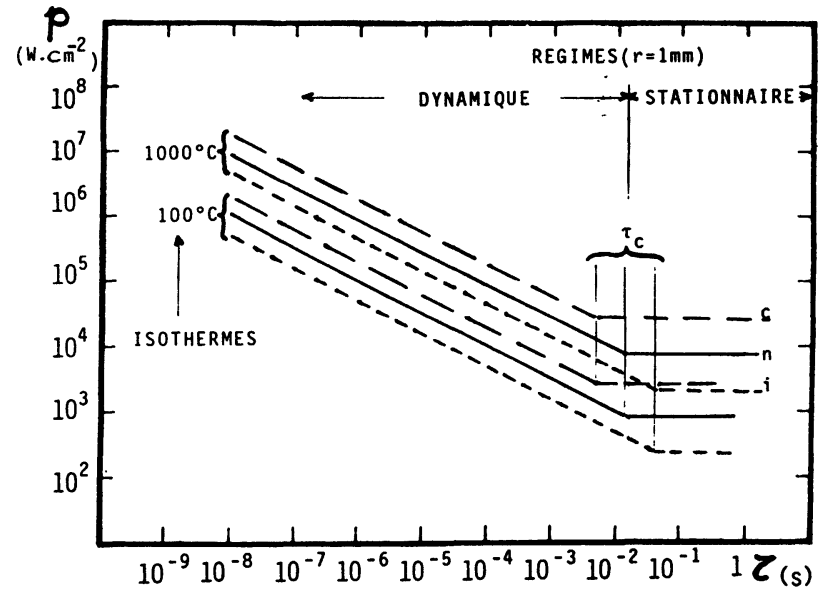

Fig. 1. - Représentation schématique de la position des isothermes 100 et $1000^{\circ} \mathrm{C}$ dans un diagramme $\log (p)-$ $\log (\tau)$ pour un faisceau de $1 \mathrm{~mm}^{2}$ de section et dans le cas de matériaux métalliques thermiquement : --- (c) « conducteurs » $(\mathrm{Cu} ., \mathrm{Al} ., \ldots),-(\mathrm{n})$ « normaux » (Fe., Aciers non alliés...), ----- (i) « isolants» (Aciers Inox, Ti., Zr., ...).

[Schematical representation of isothermal curves 100 and $1000^{\circ} \mathrm{C}$ positions in a $\log (p)-\log (\tau)$ diagram, for a $1 \mathrm{~mm}^{2}$ cross section beam. Case of metallic materials being: - - - (c) good heat conductors ( $\mathrm{Cu} ., \mathrm{Al}$.$) ,$ (n) average heat conductors (Fe., unalloyed steels, ...) ----- (i) poor heat conductors (Stainless steels, Ti., Zr., ...).]

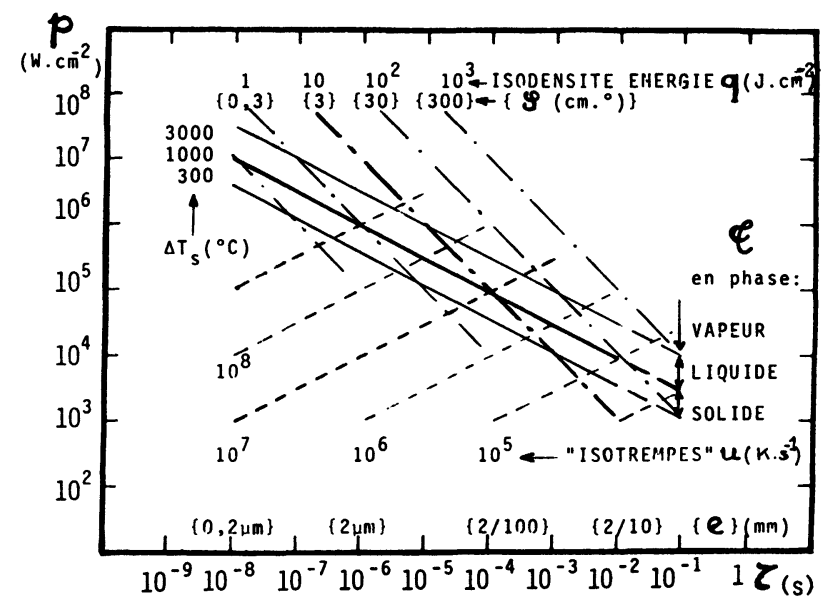

Fig. 2. - Diagramme $\log (p)-\log (\tau)$ rassemblant les différents réseaux : d'isothermes $\Delta T_{\mathrm{s}}(\longrightarrow$, d'isodensité d'énergie $q$ (— $\longrightarrow$, d'« isotrempe » $u(----)$, ainsi que les types de transformation $\mathfrak{G}$, la profondeur de pénétration $e$, et l'«intensité de transformation » $S$ qu'on est susceptible d'atteindre dans le cas d'un matériau métallique thermiquement «normal» (type acier non allié).

$\left[\log (p)-\log (\tau)\right.$ diagram collecting several curves $\Delta T_{\mathrm{s}}$ $(\stackrel{\longrightarrow}{\longrightarrow}$, isodensity of energy $q(\longrightarrow)$, isoquench $u$ (---.-), and also types of transformations $\mathcal{G}$, penetration deepness, $e$, and " intensity of transformation ", $\mathrm{S}$, which are possibly reached in the case of an average heat conductor metallic material.] $300^{\circ} \mathrm{C}$ ou $1 \mu \mathrm{m}$ à $3000^{\circ} \mathrm{C}$. Ce type de raisonnement est à la base de certaines évaluations de l'épaisseur de la couche susceptible d'être portée à l'état liquide [10].

Pour avoir une idée plus précise de l'étendue en profondeur d'une transformation microstructurale s'effectuant à l'état solide, on peut revenir sur la notion de distance de propagation de la chaleur $s \simeq 2 \sqrt{\alpha} \tau$ qui traduit en quelque sorte la distance sur laquelle s'étend le gradient thermique initial et qui permet donc d'avoir une idée de la situation des isothermes en profondeur (voir Fig. 3). La profondeur de pénétration d'une transformation microstructurale sera évidemment fonction de la température de surface $T_{\mathrm{s}}$ résultant de l'accroissement $\Delta T_{\mathrm{s}}$, du temps de traitement $\tau$ et de la température minimale $T_{\mathrm{m}}$ d'apparition de la transformation. Mais cette profondeur peut s'approximer par $e \simeq$ $2\left[\left(T_{\mathrm{s}}-T_{\mathrm{m}}\right) / \Delta T_{\mathrm{s}}\right] \sqrt{\alpha \tau}$. Cela signifie que la température de surface devra être aussi élevée que possible et que $e$ restera toujours une fraction de $\sqrt{\alpha \tau}$. Ainsi dans le cas de la trempe martensitique d'un acier $T_{\mathrm{s}}=1000^{\circ} \mathrm{C}$ et $T_{\mathrm{m}}=850^{\circ} \mathrm{C}$, d'où $e \simeq 0,3 \sqrt{\alpha \tau}$. Cette relation constitue un ordre de grandeur de l'étendue en profondeur de la plupart des transformations microstructurales à l'état solide.

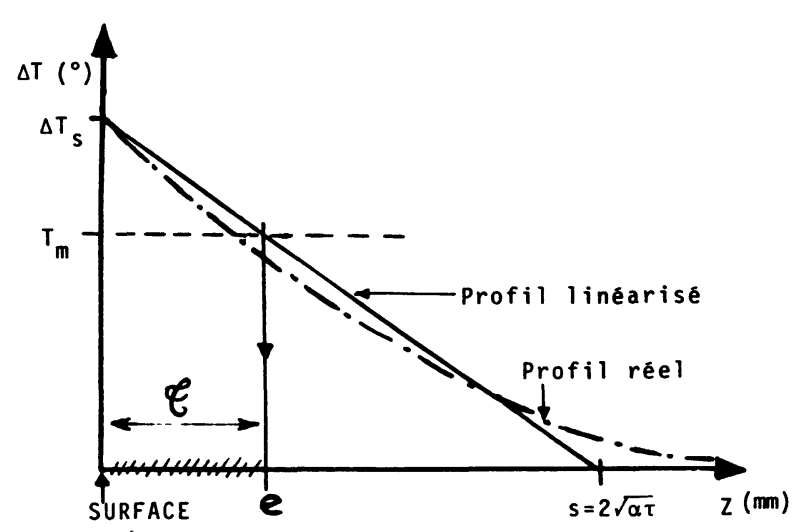

Fig. 3. - Représentation schématique de l'évolution de la température depuis la surface jusqu'à l'intérieur d'un solide avec la définition de la distance de propagation de la chaleur $s$ et de la profondeur de pénétration $e$ d'une transformation $\boldsymbol{G}$.

[Schematical representation of temperature changes from the surface to the inside of a solid, with the definition of the heat propagation length $s$ and of the penetration deepness $e$ of a transformation 6 .]

Les vitesses de montée en température et de refroidissement (trempe) constituent d'autres paramètres importants pour une transformation microstructurale. La vitesse moyenne de montée en température de surface est donnée par $U_{M}\left(\mathrm{~K} . \mathrm{s}^{-1}\right)=$ $\Delta T_{\mathrm{s}} / \tau \propto P / \sqrt{\tau}$ et les lois linéaires de la thermique [7] montrent que l'évolution de la température 
au cours du refroidissement sera assez semblable à celle durant le chauffage (voir Fig. 4) d'où $U_{R}=$ $U_{\mathrm{C}}$ ce qui permet d'avoir une idée des vitesses de trempe susceptibles d'être obtenues. Dans la représentation $\log p=f(\log \tau)$ les « isotrempes " seront des droites de pente $1 / 2$ (voir Fig. 2).

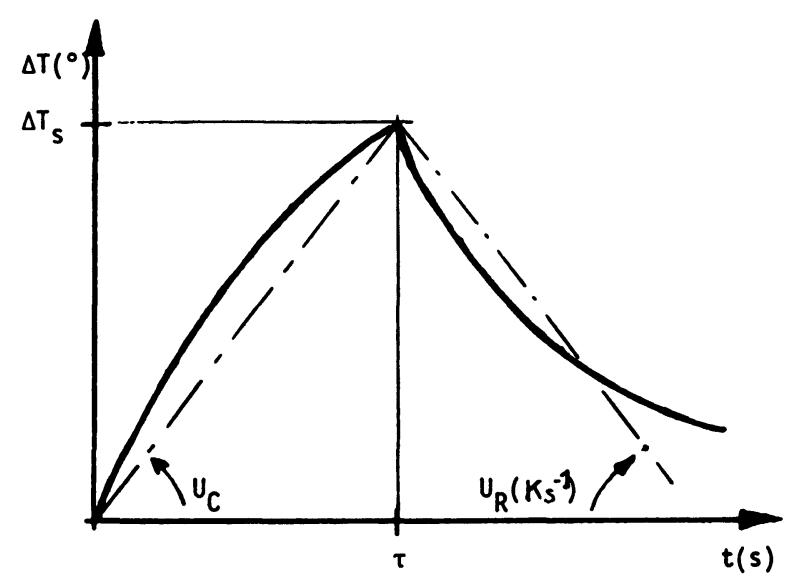

Fig. 4. - Représentation schématique de l'évolution de la température de surface d'un solide soumis à un flux laser constant durant un temps $\tau$ avec définition des vitesses moyennes de chauffage $U_{\mathrm{C}}$ et de refroidissement $U_{\mathrm{R}}$.

[Schematical representation of surface temperature changes in the case of a solid under a constant laser flux for a time $\tau$, with a definition of average rates of heating $U_{\mathrm{C}}$ and cooling $\left.U_{\mathrm{R}} \cdot\right]$

Quels que soient les alliages retenus, la démarche simplifiée que nous venons d'effectuer permet de constater qu'en définitive les comportements resteront assez semblables. Les différentes caractéristiques de la transformation recherchée (température à atteindre, temps de traitement, vitesses de chauffage et de trempe, étendue de la transformation) ne sont conditionnées que par le temps d'interaction et la densité de puissance de la source. Cette apparente simplicité recouvre en fait un certain nombre de difficultés et de restrictions d'application, les paramètres caractéristiques de la transformation ne pouvant plus être séparés comme c'est le cas pour les traitements thermiques classiques.

Par ailleurs, contrairement à ce que l'intuition conduirait à penser, l'atteinte de très hauts niveaux de densité de puissance ne présente finalement qu'un intérêt limité. En effet, soit le temps d'interaction est très court et l'étendue des effets extrêmement limitée, soit le temps d'interaction est relativement important et l'alliage se vaporisera ce qui est à éviter en traitement thermique. Les sources laser pulsées délivrant de hautes puissances durant des temps courts $\left(10^{-9} \mathrm{~s}\right.$ ou moins) ne sont donc pas spécialement intéressantes en traitement thermique, d'autant plus que les effets ne sont pas simplement additifs. Ainsi, une succession d'impulsions peut fondre puis resolidifier alternativement le même petit volume de matière. En conséquence, les valeurs moyennes de la puissance communiquée peuvent être sans signification quant à l'étendue de la transformation observée.

\section{Les différentes catégories de traitements thermi- ques réalisables par laser.}

Les considérations que nous avons dégagées dans les deux paragraphes précédents permettent déjà de prévoir que seuls les traitements thermiques de type superficiel réalisés en vue d'obtenir des états microstructuraux hors équilibre peuvent être réalisés valablement avec des sources laser.

Nous allons préciser cela en envisageant successivement les transformations s'effectuant à l'état solide puis celles nécessitant le passage à l'état liquide.

4.1 TRANSFORMATIONS À L'ÉTAT SOLIDE. - Le but des traitements thermiques entrant dans cette catégorie est, partant d'un état stable à haute température :

- soit de le figer par refroidissement rapide : hypertrempe, par exemple cas de la «trempe » des alliages légers à durcissement structural,

- soit de donner naissance à un état structural hors équilibre, par transformation allotropique : par exemple cas de la trempe martensitique des aciers au carbone et faiblement alliés.

Pour ces deux types de traitements on doit tout d'abord obtenir à haute température une solution solide homogène, ce qui nécessite l'activation de processus de diffusion atomique, puisque l'état de départ des alliages est généralement multiphasé. Selon les couples soluté-solvant les coefficients de diffusion $D$ peuvent être très variables mais de toute façon ils suivent des relations du type $D=$ $D_{0} \exp (-Q / R T)$ et les distances de diffusion peuvent être appréciées à partir de $d=2 \sqrt{D \tau}$. Cellesci doivent être au moins de l'ordre de grandeur des distances séparant les particules contenues dans une matrice pour que l'homogénéisation puisse être réalisée. Or, même à des températures proches de la fusion de l'alliage, $D$ reste généralement de l'ordre de $10^{-6}-10^{-8} \mathrm{~cm} \cdot \mathrm{s}^{-1}$ [11]. Les distances inter-particules ne doivent donc pas dépasser 1 à $10 \mu \mathrm{m}$ pour des temps d'interaction de l'ordre de $1 \mathrm{~s}$ et 10 à $100 \mathrm{~nm}$ pour des temps d'interaction de l'ordre de $10^{-4} \mathrm{~s}$. Cela entraîne trois conséquences :

1) Des traitements thermiques nécessitant des transformations diffusionnelles en phase solide sont pratiquement irréalisables pour des temps d'interaction inférieurs à $10^{-3} \mathrm{~s}$.

2) L'état microstructural initial aura une très grande importance et devra toujours être constitué 
d'un agrégat de phases dispersées le plus finement possible.

3) Les températures maximales atteintes devront être les plus hautes possible et sensiblement plus élevées que pour les traitements classiques.

Il s'ensuit que la mise en solution avant trempe d'alliages légers est peu réaliste ( $D$ ne pouvant guère dépasser $10^{-9} \mathrm{~cm}^{2} \cdot \mathrm{s}^{-1}$ ), et que seuls des traitements de type austénitisation avant trempe martensitique peuvent être pratiqués (l'intervalle de température d'austénitisation est suffisant pour atteindre des coefficients $D \simeq 10^{-6}-10^{-7} \mathrm{~cm}^{2} \cdot \mathrm{s}^{-1}$ ) [12].

Pour réaliser une trempe martensitique, outre l'austénitisation il est ensuite nécessaire d'avoir des vitesses de refroidissement suffisamment élevées mais qui excèdent rarement $10^{3} \mathrm{~K} \cdot \mathrm{s}^{-1}$ même pour des alliages peu trempants. Cette condition est donc particulièrement facile à remplir et il sera ainsi possible d'obtenir des transformations martensitiques sur des alliages réputés «non trempants » (aciers doux, entre autres).

La profondeur de trempe minimale désirée par les utilisateurs dépasse généralement $0,1 \mathrm{~mm}$, ce qui implique là encore des temps d'interaction ne pouvant pas descendre en dessous de $10^{-3} \mathrm{~s}$.

Par conséquent, le domaine représentatif des traitements de trempe martensitique dans les aciers, se situe dans le diagramme $\log p=f(\log \tau)$ dans une étroite bande centrée autour de l'isotherme de surface $1000^{\circ} \mathrm{C}\left( \pm 100^{\circ}\right.$ environ) est délimitée par les temps $10^{-3}$ et $1 \mathrm{~s}$ (voir Fig. 5 , où sont rassemblés

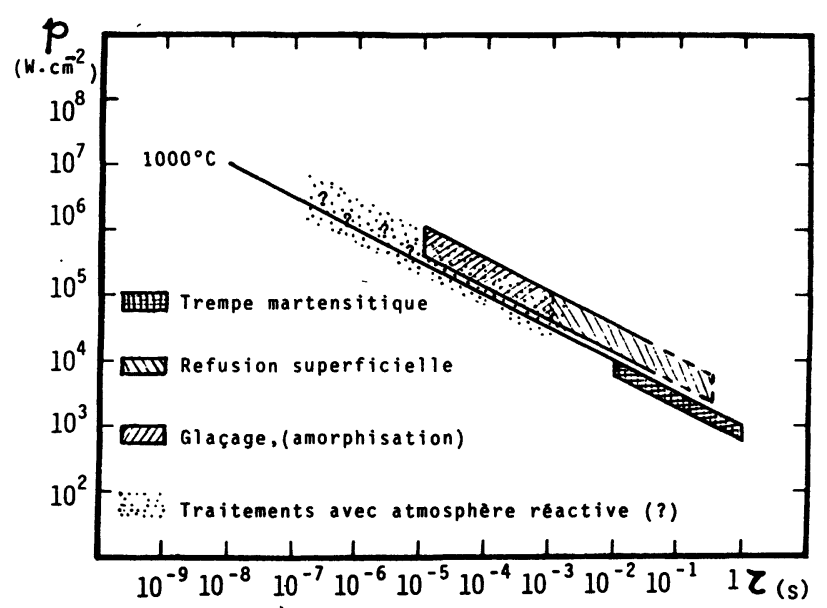

Fig. 5. - Diagramme $\log (p)-\log (\tau)$ correspondant aux différentes catégories de transformations microstructurales susceptibles d'être réalisées sur les alliages ferreux (ce diagramme reste en fait valable en première approximation pour les autres familles d'alliages métalliques).

$[\log (p)-\log (\tau)$ diagram corresponding to the different microstructural transformations susceptible of realization on ferrous alloys (this diagram is in first approximation still valid for other families of metallic alloys).] les différents traitements susceptibles d'être effectués sur les alliages ferreux). On remarquera que cette figure est conforme aux nombreux diagrammes établis empiriquement (voir par exemple $[13,14]$ ) et qui sont souvent désignés « diagramme d'Engel» sans que l'origine physique en soit pressentie.

4.2 TRANSFORMATIONS A PARTIR DE L'ÉtAT LIQUIDE. - Les traitements thermiques entrant dans cette catégorie consistent généralement à partir d'un alliage à l'état liquide et à le refroidir suffisamment rapidement pour que :

- soit sa solidification se fasse avec des grains (ou des lamelles de phases) les plus fins possible : cas en particulier des alliages légers de fonderie,

- soit sa solidification s'effectue en ne suivant plus le diagramme d'équilibre : cas en particulier des fontes grises sur lesquelles on veut une couche superficielle de fonte blanche,

- soit on refroidit suffisamment rapidement pour hypertremper l'état liquide et donc obtenir un matériau amorphe : cas des verres métalliques.

De toute façon quel que soit le cas envisagé, il faut porter les couches superficielles de l'alliage à l'état liquide, mais évidemment sans atteindre sa vaporisation. Cette fois, contrairement aux transformations à l'état solide, les problèmes d'homogénéisation sont beaucoup moins cruciaux du fait de l'augmentation de la diffusion et des courants de convection dans le liquide. Du point de vue niveau de densité de puissance nécessaire, outre la température recherchée dans le bain (de l'ordre de 1000 à $2000^{\circ} \mathrm{C}$ en général) il faut prendre en compte la chaleur latente de fusion qui équivaut énergétiquement à un accroissement de température de 500 à $1000^{\circ} \mathrm{C}$ (cf. § 2). Dans notre diagramme, ce type de traitement nécessite donc de se situer au niveau des «isothermes » $1500-3000{ }^{\circ} \mathrm{C}$.

Dans le cas des fontes, des vitesses de refroidissement de l'ordre de $10^{2}-10^{3} \mathrm{~K} . \mathrm{s}^{-1}$ suffisent pour passer du diagramme stable Fe-graphite au diagramme métastable $\mathrm{Fe}-\mathrm{Fe}_{3} \mathrm{C}$ et donc former une couche de fonte blanche; des temps d'interaction de l'ordre de $1 \mathrm{~s}$ conviennent donc parfaitement et seule l'épaisseur de la couche durcie désirée limitera la diminution du temps d'interaction [15].

Pour les alliages légers de fonderie, on cherche généralement à avoir des lamelles eutectiques les plus fines possible et des vitesses de refroidissement de $10^{4}-10^{5} \mathrm{~K} . \mathrm{s}^{-1}$ sont nécessaires; le domaine de temps d'interaction utilisable se trouve donc centré entre $10^{-1}$ et $10^{-2} \mathrm{~s}$ et l'épaisseur des couches ainsi refondues sera de l'ordre de quelques dixièmes de $\mathrm{mm}[16]$.

L'obtention de «verres métalliques » outre la nécessité de partir d'alliages de composition appropriée impose des vitesses de refroidissement généralement comprises entre $10^{5}$ et $10^{7} \mathrm{~K} . \mathrm{s}^{-1}$, donc des 
temps d'interaction dans la gamme $10^{-3}-10^{-5} \mathrm{~s}$, voire inférieurs, mais évidemment les épaisseurs susceptibles d'être ainsi amorphisées seront d'autant plus faibles et ne pourront guère dépasser quelques dizaines de $\mu \mathrm{m}$ [17].

Sans atteindre l'état amorphe, il est quand même possible d'obtenir des états extrêmement hors équilibre en réalisant des traitements dans le domaine repéré «zone de glaçage » sur notre diagramme (Fig. 5). Cette zone fait immédiatement suite à la zone des traitements de «refusion superficielle» relative aux fontes, qu'elle chevauche partiellement.

\section{Remarques :}

1) Bien que non exhaustive cette revue des traitements couvre à peu près l'ensemble des situations envisageables [18]. Il en ressort que « la fenêtre » définissant les paramètres d'utilisation est assez étroite.

En particulier, si des temps d'interaction inférieurs à $10^{-5} \mathrm{~s}$ sont certainement susceptibles d'induire des transformations hors équilibre dans les couches superficielles de pièces irradiées, il est toutefois peu concevable à l'heure actuelle que le traitement ainsi réalisé permette des applications dans le domaine de la mécanique, à moins que cette irradiation ne se fasse en présence d'une atmosphère particulière susceptible d'interagir avec le rayonnement [19] et par voie de conséquence de modifier la composition chimique des couches superficielles du matériau et peut-être certaines propriétés telles que la tenue à la corrosion (traitements à rapprocher de certains pratiqués pour la réalisation de composants en microélectronique-c.v.d. [20]).

2) $\mathrm{Si}$ le temps d'interaction est de l'ordre de $10^{-9} \mathrm{~s}$, on arrive au stade où la distance de propagation de la chaleur devient du même ordre de grandeur que la distance de pénétration du rayonnement infrarouge incident, la température s'élève très vite et provoque la vaporisation des couches superficielles, d'où création d'un plasma et développement d'ondes de choc susceptibles de modifier l'état microstructural du matériau - traitement dit de durcissement par impulsion [21]. Ceci sera obtenu par un faisceau laser mais sera un traitement thermomécanique plus qu'un réel traitement thermique.

\section{Conception d'une installation laser pour traite- ments thermiques.}

Nous avons défini les caractéristiques théoriques que doit présenter une source laser pour permettre la réalisation de traitements sur des matériaux à usage mécanique ainsi que les limitations inhérentes à l'utilisation du laser au niveau des modifications induites dans le matériau, nous allons maintenant chercher à préciser, d'un point de vue plus technique les conséquences de ces considérations.
Jusqu'à maintenant on a évoqué un temps d'interaction $\tau$ entre le rayonnement et le matériau sans préciser sa définition. Selon la nature de la source deux situations sont à envisager :

- soit le laser travaille en régime pulsé et le temps d'interaction peut être assimilé à la durée de l'impulsion ; tous les raisonnements effectués auparavant se trouvent intégralement conservés, la périodicité d'émission des impulsions étant généralement grande vis-à-vis de leur durée. Il se pose cependant le problème de la continuité dans la réalisation du traitement, une juxtaposition de pulses entraînant presque toujours une inhomogénéité dans la transformation réalisée. Ce type de source est donc pour le moment assez peu utilisé en traitement thermique,

- soit le laser émet en continu et le temps d'interaction ne peut alors être défini qu'à partir d'un mouvement relatif entre la pièce et le faisceau. Ce mouvement s'effectuant à la vitesse $v\left(\mathrm{~mm} . \mathrm{s}^{-1}\right)$, le temps d'interaction peut être assimilé au temps d'interaction équivalent $\tau^{+}=r_{x} / v$, avec $r_{x}$ paramètre représentatif de la dimension du faisceau dans la zone d'interaction faisceau-matériau et selon la direction du déplacement relatif.

Il faut insister sur la définition de cette « dimension de faisceau » qui doit être capable de caractériser une répartition d'énergie et d'en prévoir les effets; or, pour le moment, il règne la plus grande confusion dans ce domaine. Ainsi, pour une répartition gaussienne $r$ peut être défini par la dimension prise à $1 / e, 1 / e^{2}$ voire $1 / e^{3}[8,9]$ ou encore dimension à mi-hauteur et le problème se complique pour des répartitions réelles plus complexes.

Etant donné que les effets induits sont pour l'essentiel conditionnés par la quantité d'énergie transportée et par la densité maximale de puissance, à notre avis la manière la plus simple de décrire une répartition d'énergie quelconque serait encore d'introduire la notion de rayon équivalent $r^{+}$, ce rayon étant défini comme le rayon du faisceau cylindrique équivalent ayant une densité de puissance constante égale à la valeur maximale de la répartition considérée et transportant la même quantité d'énergie. Par exemple dans le cas d'une répartition gaussienne, $r^{+}$correspondra à la dimension du faisceau pris à $1 / e$ de la hauteur de la gaussienne et le rayon à $1 / e^{2}$ généralement utilisé par les opticiens vaudra $\sqrt{2} r^{+}$(voir Fig. 6).

Outre la difficulté de définition et de détermination expérimentale de la dimension du faisceau, par son intermédiaire les paramètres $p$ et $\tau$ sont maintenant dépendants. Il n'est plus possible d'utiliser les simples diagrammes $\log p-\log \tau$, et il faut leur substituer les diagrammes isothermes $P-v-r$ pour choisir les paramètres de traitement susceptibles d'induire une transformation donnée, et la condition $\tau<r^{2} / 4 \alpha$ doit être remplacée par la condition 


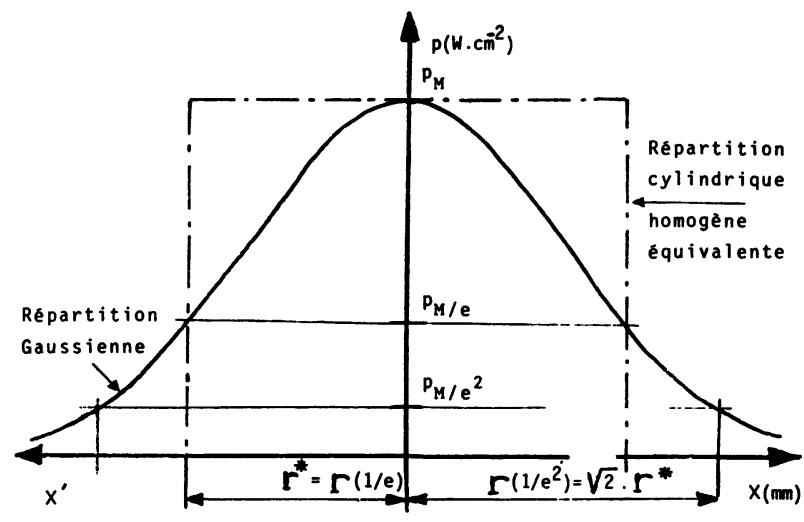

Fig. 6. - Représentation schématique de la répartition d'énergie pour un faisceau gaussien de rayon $r$ à $1 / e^{2}$ avec définition du rayon équivalent $r^{+}$à partir de la répartition cylindrique homogène $\left(r^{+}=0,707 r\right)$.

[Schematical representation of the energy distribution for a Gaussian beam with a $1 / e^{2}$ radius $r$ and the definition of the equivalent radius $r^{+}$from the equivalent cylindrical homogenous distribution $\left(r^{+}=0,707 r\right)$.]

$v>4 \alpha / r$ (voir par exemple la Fig. 7 correspondant à l'atteinte de la fusion de l'aluminium) [22].

Il faut également remarquer que les isochrones $\tau^{+}$sont alors constituées par un réseau de courbes de pente 1 dans le régime stationnaire et 2 dans le régime dynamique.

D'un point de vue plus pratique la reproductibilité d'un traitement et son homogénéité seront fonction de la densité d'énergie réellement absorbée par unité de temps à chaque instant et en chaque point

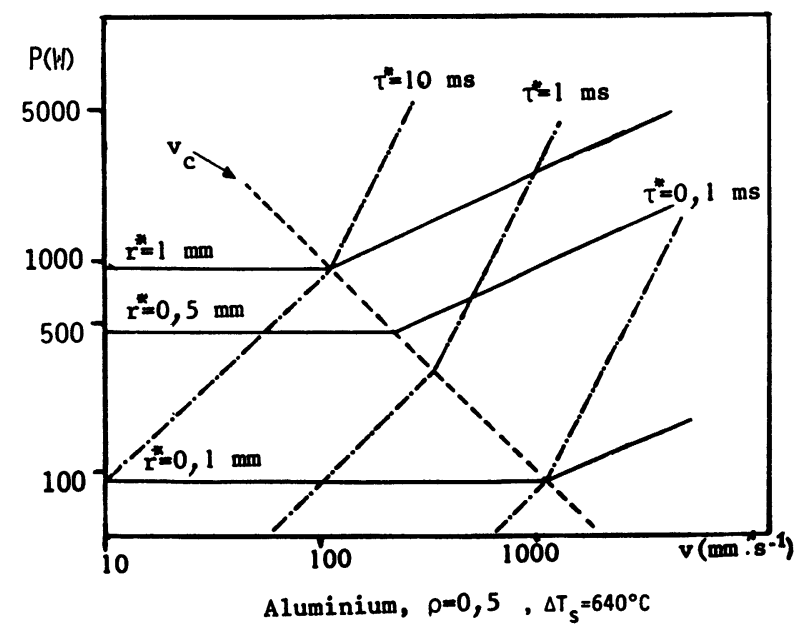

Fig. 7. - Exemple de diagramme isotherme $P-v-r$ indiquant les différents groupes de paramètres de traitement conduisant à l'atteinte de la température de fusion de l'aluminium, ainsi que le temps d'interaction laser-matériau correspondant.

[An example of an isothermal $P$-v $-r$ diagram pointing out several groups of parameters leading to the melting of aluminium, and the corresponding interaction time.] d'un matériau. Pour réaliser des traitements thermiques de qualité, il est nécessaire de pouvoir corriger et moduler la répartition d'énergie du faisceau incident, donc que :

1) la cavité ait une émission stable dans le temps, en puissance et en répartition d'énergie (stabilité du mode d'émission),

2) la répartition d'énergie soit constante ou évolue selon des lois simples lorsque la puissance moyenne transportée est modifiée,

3) la répartition d'énergie soit caractérisable, et cela si possible en temps réel, afin de pouvoir éventuellement modifier en cours de traitement certains paramètres de la source pour corriger une anomalie.

Ces conditions étant au moins partiellement remplies, il faudrait également mettre au point des optiques permettant la transformation de la répartition énergétique. Différents dispositifs à miroirs oscillants ou multi-miroirs avec superposition d'images ont déjà été conçus et certains d'entre eux testés; des dispositifs utilisant l'holographie sont également concevables. De toute façon la répartition idéale pour traiter une surface plane correspond à un faisceau homogène ayant des fluctuations de répartition d'énergie du même ordre de grandeur que les fluctuations de température acceptables (5\% environ en pratique). De plus cette répartition devra présenter deux flans nets perpendiculaires à la direction de déplacement (voir Fig. 8) [23, 24].

Une fois le faisceau «mis en forme » et amené au niveau de la pièce à traiter la localisation de la zone d'interaction et la modulation du temps d'interaction pour les sources émettant en continu imposent l'emploi de tables à commande numérique. Un

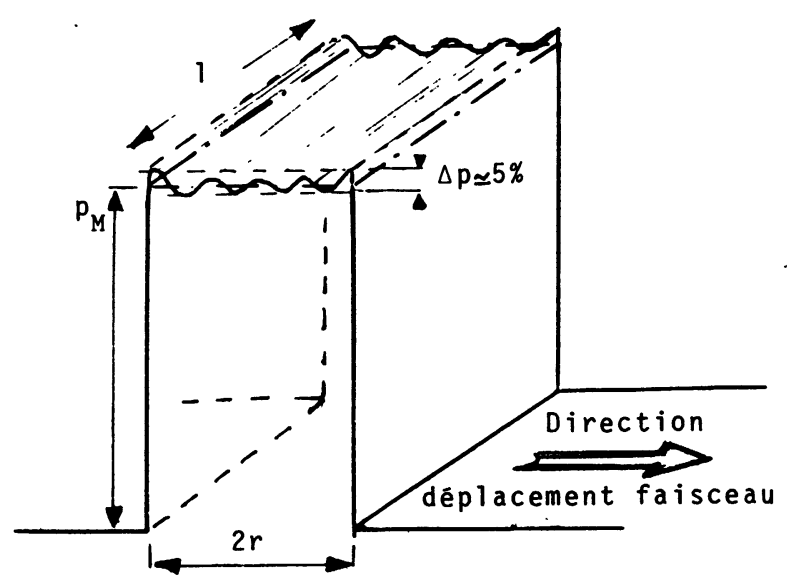

Fig. 8. - Représentation schématique de la répartition d'énergie idéale permettant le traitement homogène de surface.

[Schematical representation of the ideal energy distribution allowing a homogenous surface treatment.] 
traitement thermique sur une pièce de forme plus ou moins complexe nécessiterait en outre de tenir compte de ses dimensions géométriques, de son échauffement progressif au cours du traitement et des modifications éventuelles des conditions d'absorption du rayonnement. Il serait donc nécessaire de suivre l'avancement en temps réel de la transformation recherchée, donc d'avoir accès à un paramètre corrélable avec celle-ci, le plus simple étant sans doute encore l'évolution de la température de surface, mais des mesures beaucoup plus sophistiquées peuvent être envisagées (radiographie X-spectrographie-techniques ultrasonores [25]). Le suivi de tels paramètres devrait permettre, après traitement informatique, de modifier les paramètres de commande de la source (et éventuellement de l'optique de transport et de mise en forme).

Notons enfin que dans la zone d'interaction, l'atmosphère gazeuse ambiante joue un très grand rôle vis-à-vis de l'oxydation, mais intervient également au niveau de la conversion énergétique qui peut être modifiée considérablement selon les caractéristiques du plasma formé et les couches d'oxydes développées. Ce point ne doit donc pas être négligé si l'on veut maîtriser un traitement thermique [10].

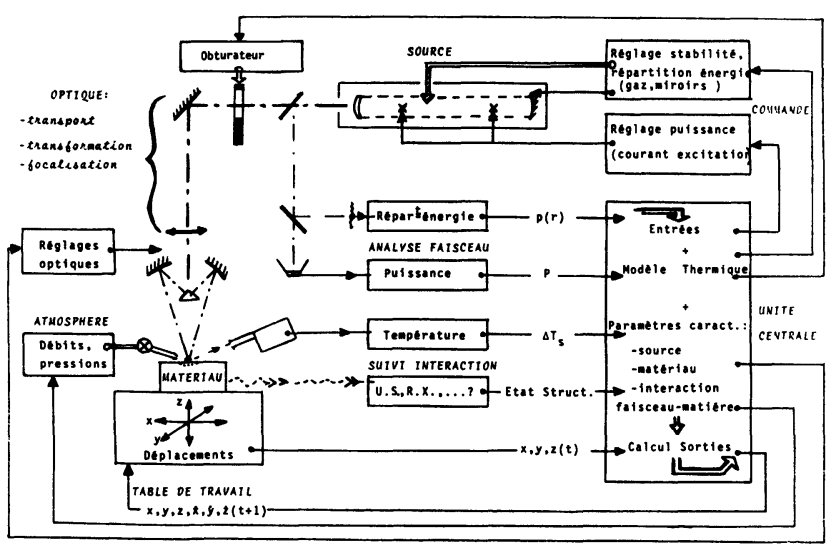

Fig. 9. - Organigramme de l'installation laser de traitement thermique idéale.

[Organization chart of an ideal laser plant for heat treatment.]

L'organigramme correspondant à l'installation laser «idéale » pour la réalisation de traitements thermiques est présenté figure 9.

\section{Conclusions.}

Si les possibilités du laser dans le domaine du traitement thermique des alliages sont incontestables, il faut cependant prendre conscience que la seule existence d'une source laser ne suffit pas pour en faire une installation performante ; en particulier si des sources émettant plus de $10 \mathrm{~kW}$ moyens sont intéressantes, il est encore plus urgent de réaliser des sources de très grande stabilité d'émission (et c'est encore malheureusement rarement le cas dès que l'on dépasse le $\mathrm{kW}$ ) et de grande fiabilité. Il est également nécessaire qu'une installation ne se limite pas à la source et la table de travail mais intègre optique de transport et de transformation du faisceau ainsi qu'un système de métrologie temps réel de celui-ci et des effets induits permettant par l'intermédiaire d'un calculateur central d'asservir le système et d'agir sur les paramètres de commande de la source.

De telles installations sont d'une complexité nettement supérieure à celles nécessaires pour l'utilisation en découpe et même en soudage ; cependant, il y a déjà cinq ans, une installation répondant aux spécifications définies précédemment était proposée sur le marché avec en particulier mise en forme du faisceau, asservissement de la puissance et de la température de surface d'échantillon [26].

Il sera également nécessaire d'accroître les connaissances au niveau du comportement des matériaux vis-à-vis de l'absorption du rayonnement, du comportement thermique et des évolutions microstructurales dans des conditions hors d'équilibre bien plus extrêmes que celles envisagées habituellement par les métallurgistes. Des diagrammes reliant les paramètres de traitement $(P, v, r$, atmosphère, revêtement absorbant), aux effets induits dans le matériau ( $T$, évolution microstructurale, état de contrainte, profondeur durcie, etc.) devront être établis de manière à avoir l'équivalent des diagrammes T.T.T. ou T.R.C. indispensables pour la réalisation correcte de traitements thermiques classiques.

Par ailleurs la technologie laser dans le domaine des traitements thermiques est certainement plus intéressante par les perspectives nouvelles qu'elle offre (localisation, modulation du traitement, atteinte d'états microstructuraux hors équilibre) que par sa simple substitution aux technologies classiques. Il faudra certainement repenser matériaux et pièces selon leurs fonctions et les possibilités de ces nouvelles technologies que constituent le laser et les faisceaux de haute énergie d'une manière générale (faisceaux d'électrons et implantation ionique) et aboutir ainsi à la notion de pièces ou de matériaux à structure et propriétés composites [27], un alliage de base aux performances modestes voyant sa composition et ses propriétés modulées selon les sollicitations de la pièce.

Enfin, pour conclure, nous citerons Cohen et Epperson [10] qui écrivaient il y a déjà vingt ans : « Nous insistons sur le fait que si l'on peut d'ores et déjà utiliser avantageusement le laser sans bien comprendre la nature des phénomènes qui interviennent durant l'interaction avec le matériau, le laser deviendra un outil encore bien plus performant s'il 
est utilisé avec toutes les précautions et la maîtrise qui ne peuvent résulter que d'une compréhension des mécanismes mis en jeu ».

Nous ajouterons seulement que la maîtrise de cette technologie dans le domaine des traitements thermiques passe aussi par le suivi en temps réel des caractéristiques des sources et des effets résultant de l'interaction faisceau-matériau, ce qui était irréaliste il y a encore dix ans et explique peut-être ces années de lente évolution.

\section{Bibliographie}

[1] Metals Handbook (8th ed.) (A.S.M.), 1961, p. 44.

[2] Touloukian, Y. S. and DEWITT, D. P., Thermophysical Properties of Matter (Plenum, New York), 1972.

[3] Lasers Applications Chart, Lasers \& Applications (High Tech. Publications Inc.), 1986.

[4] ENGEL, S. L., Society of Manufacturing Engineers, Technical Paper No MR 76-857 (1976).

[5] Bloembergen, N., Appl. of Lasers in Material Processing (A.S.M.) 1979, p. 1-11.

[6] Sanders, D. J., Appl. Opt. 23 (1984) 30-35.

[7] Carslaw, H. S. and Jaeger, J. C., Conduction of Heat in Solids (Oxford U.P., 2nd ed.) 1959.

[8] Pittaway, L. G., Br. J. Appl. Phys. 15 (1964) 967982.

[9] Hsu, S. C., Kou, S. and Mehrabian, R., Metal. Trans. B 11 (1980) 29-38.

[10] Cohen, M. I. and Epperson, J. P., Electron Beam and Laser Beam Technology, ed. L. Marton and A. B. El-Kareh (Academic Press, New York), 1968, p. 139-186.

[11] Philibert, J., Diffusion et Transport de Matière dans les Solides (Editions de Physique, Paris), 1985.

[12] Ashby, M. F. and EAsterling, K. E., Acta Metall. 32 (1984) 1935-1948.

[13] Allan, R., I.E.E.E. Spectrum 16 (1979) 42-49.

[14] Carstens, J. P., Material Processing with Multikilowatt Lasers, United Tech. Res. Center, Oct. 1976.

[15] Tucker, T. R., Clauer, A. H. and McCall, J. L. Microstruct. Sci. 10 (1982) 347-358.
[16] Anisovitch, G. A., Chatourov, G., Pavlenko, E. and NaOUTCHENKo, N., Isv. Akad. Nauk BSSR, Ser. Fiz.-Tekh. Nauk 1 (1979) 57-65.

[17] von Allmen, M., Top. Appl. Phys. 53 (1983) 261281.

[18] Gnanamuthu, D. S., Appl. Lasers Material Processing (A.S.M.) 1979, p. 177-211.

[19] Walker, A., Folkes, J., Steen, W. M. and West, D. R. F., Surf. Eng. 1 (1985) 185-201.

[20] BÄUerle, D., Laser Processing and Diagnostics (Springer Verlag, Berlin), 1984.

[21] Clauer, A. H., Fairand, B. P. and Slater, J. E., rapport N.A.S.A. CR-145132, Feb. 1977.

[22] Dietz, J. et Merlin, J., Process Problèmes Thermiques Liés à l'Utilisation des Lasers (Société Française des Thermiciens, Paris), 21 mai 1986 (à paraître).

[23] Kawasumi, H., Source Book on Appl. of the Laser in Metalworking (A.S.M.) 1981, p. 201-205.

[24] Li, J. C., Merlin, J. et Perez, J., Revue Phys. Appl. 21 (1986) 425-433.

[25] Rawlings, R. D. and Steen, W. M., Opt. Lasers Eng. 2 (1981) 173-188.

[26] Feinberg, R. M., A.V.C.O. Everett H.P.L. Lasers Technical Notice, 1981.

[27] Merlin, J. and Dietz, J., Proceedings of Aluminium Technology'86 (The Institute of Metals, London) 1986 , p. 87.1-87.4. 